\title{
Video Article \\ Basic Surgical Techniques in the Göttingen Minipig: Intubation, Bladder Catheterization, Femoral Vessel Catheterization, and Transcardial Perfusion
}

\author{
Kaare S. Ettrup ${ }^{1,2}$, Andreas N. Glud ${ }^{2}$, Dariusz Orlowski ${ }^{2}$, Lise M. Fitting ${ }^{1}$, Kaare Meier ${ }^{1}$, Jens Christian Soerensen ${ }^{1}$, Carsten R. Bjarkam ${ }^{1,2}$, Aage K. $^{2}$ \\ Olsen Alstrup ${ }^{3}$ \\ ${ }^{1}$ Department of Neurosurgery, Aarhus University Hospital \\ ${ }^{2}$ Department of Neurobiology, Institute of Anatomy, Faculty of Health Sciences, Aarhus University \\ ${ }^{3}$ Positron Emission Tomography (PET) Centre, Aarhus University Hospital
}

Correspondence to: Kaare S. Ettrup at kse@ana.au.dk

URL: https://www.jove.com/video/2652

DOI: doi:10.3791/2652

Keywords: Medicine, Issue 52, Animal model, Animal preparation, Animal handling, Sus scrofa, Swine, Tissue fixation

Date Published: 6/26/2011

Citation: Ettrup, K.S., Glud, A.N., Orlowski, D., Fitting, L.M., Meier, K., Soerensen, J.C., Bjarkam, C.R., Alstrup, A.K. Basic Surgical Techniques in the Göttingen Minipig: Intubation, Bladder Catheterization, Femoral Vessel Catheterization, and Transcardial Perfusion. J. Vis. Exp. (52), e2652, doi:10.3791/2652 (2011).

\section{Abstract}

The emergence of the Göttingen minipig in research of topics such as neuroscience, toxicology, diabetes, obesity, and experimental surgery reflects the close resemblance of these animals to human anatomy and physiology ${ }^{1-6}$. The size of the Göttingen minipig permits the use of surgical equipment and advanced imaging modalities similar to those used in humans ${ }^{6-8}$. The aim of this instructional video is to increase the awareness on the value of minipigs in biomedical research, by demonstrating how to perform tracheal intubation, transurethral bladder catheterization, femoral artery and vein catheterization, as well as transcardial perfusion.

Endotracheal Intubation should be performed whenever a minipig undergoes general anesthesia, because it maintains a patent airway, permits assisted ventilation and protects the airways from aspirates. Transurethral bladder catheterization can provide useful information about about hydration state as well as renal and cardiovascular function during long surgical procedures. Furthermore, urinary catheterization can prevent contamination of delicate medico-technical equipment and painful bladder extension which may harm the animal and unnecessarily influence the experiment due to increased vagal tone and altered physiological parameters. Arterial and venous catheterization is useful for obtaining repeated blood samples and monitoring various physiological parameters. Catheterization of femoral vessels is preferable to catheterization of the neck vessels for ease of access, when performing experiments involving frame-based stereotaxic neurosurgery and brain imaging. When performing vessel catheterization in survival studies, strict aseptic technique must be employed to avoid infections ${ }^{6}$. Transcardial perfusion is the most effective fixation method, and yields preeminent results when preparing minipig organs for histology and histochemistry, ${ }^{2,9}$. For more information about anesthesia, surgery and experimental techniques in swine in general we refer to Swindle 2007. Supplementary information about premedication and induction of anesthesia, assisted ventilation, analgesia, pre- and postoperative care of Göttingen minipigs are available via the internet at http://www.minipigs.com ${ }^{10}$. For extensive information about porcine anatomy we refer to Nickel et al. Vol. 1-5 $5^{11}$.

\section{Video Link}

The video component of this article can be found at https://www.jove.com/video/2652/

\section{Protocol}

\section{Intubation}

1. Equipment needed for endotracheal intubation: means of assisted ventilation, slings for opening the mouth, low pressure suction with blunt tip, veterinary laryngoscope with straight blade between 17 and $25 \mathrm{~cm}$ long, stylet, syringe with air, stethoscope and adhesive tape. For adult Göttingen minipigs an endotracheal tube size 5 to $7 \mathrm{~mm}$ is appropriate ${ }^{10}$. In this video a $5.5 \mathrm{~mm}$ tube was used ${ }^{10}$.

2. The minipig is placed on its back and properly supported in order to ensure that the laryngeal passage is kept straight.

3. The tongue is pulled out slightly and the jaw held open by an assistant without overextending the head. Rotation of the airways or excessive extension of the head can occlude airways, making the laryngeal opening more difficult to identify and complicating tracheal placement of the tube.

4. The laryngoscope is passed into the pharyngeal cavity and used to depress the tongue, making the epiglottis visible. The tip of the suction is used to displace the epiglottis from the soft palate. The tip of the laryngoscope is then used to press the epiglottis upward toward the base of the tongue, revealing the laryngeal opening.

5. The arytenoids cartilages and vocal cords come into view and the endotracheal tube is advanced gently with a slight rotation, into the trachea during expiration.

6. The tube cuff is inflated according to the specifications of the manufacture while taking care to avoid excess inflation, which may cause swelling and edema of the airways. Pigs have a bronchus to ventilate the right cranial pulmonal lobe. It is therefore important to place the 
tube above the tracheal bifurcation. This is facilitated by using the correct tube size and importantly by fastening the proximal end of the tube at the level of the snout using adhesive tape.

7. When the minipig is properly intubated, a free passage of air must be felt through the tube, and chest auscultation should reveal respiration sounds in both the left and right side of the thorax.

8. Upon connecting the ventilator with monitoring system to the minipig, physiological expiratory carbon dioxide values should be measurable and the carbon dioxide curve that follows the respiration pattern decided by the ventilator settings ${ }^{6,10}$ can be observed.

9. The carbon dioxide and oximetry values may initially be affected by the premedication and anesthesia induction. However these values should quickly revert back to normal with proper ventilation ${ }^{6,10}$. Any sign of cyanosis despite artificial-assisted ventilation indicate improper placement of the tube. The most common error is inadvertent placement of the tube in the esophagus.

\section{Transurethral bladder catheterization}

1. This procedure is only for female pigs. For adult minipigs a Foley catheter French size 8 or 10 is appropriate. Due to the curvature and size of the external urethral opening, a catheter with stylet is preferable. A nasal speculum can be used in small minipigs whereas a speculum with longer blades can be useful for large animals. Necessary equipment also includes a blunt-tip forceps, a syringe with saline, lubricant and a closed system urine drainage bag.

2. In order to best visualize the external urethral orifice during catheterization the minipig is placed on its back with a support under the lumbar region, while an assistant pulls the hind legs cranially.

3. The lubricated speculum is inserted and opened to visualize the vaginal wall. The female external urethral opening is located in the floor of the vagina approximately one-third to one-half of the distance to the cervix (Fig. 1A). However, because the animal is placed on the back, the position of the urethral opening will appear to be inverted.

4. The catheter is controlled with the blunt-tip forceps, so the tip of the catheter is in continuation of the forceps. Carefully insert the catheter in the urethral opening $\left({ }^{\text {Fig. } 1 \mathrm{~B}}\right)$. The urethra is easily traumatized if too much force is used during catheterization. However, slight resistance may initially be felt until the catheter has passed the external urinary sphincter. If this problem persists, this may indicate that the animal is not sedated deeply enough. The solution is to wait awhile and let the anesthesia take its effect.

5. As the catheter reaches the bladder, remove the stylet, and urine will be seen flowing.

6. Inflate the balloon with saline according to the specifications of the manufacture and gently pull the catheter out until the balloon catches on the bladder neck.

7. Attach the closed-system urine drainage bag

8. Tape the catheter to the tail to avoid accidental displacement of the catheter when handling the animal.

\section{Femoral artery and vein catheterization}

1. Necessary equipment includes: scalpel, blunt-tip surgical scissors, tissue forceps, small blunt-tip surgical forceps, small self-retaining tissue retractor, needle holder, surgical swaps, suture with needle, heparinized saline as a flush-fluid to maintain catheter patency, two 18-gauge IV cannula, two 4-French brite tip sheaths with introducer and Seldinger guide wire.

2. The depth of anesthesia should be assessed by testing the interdigital pain reflex before initiating the procedure.

3. The femoral artery and vein are approached with the minipig placed on its back and the rear leg retracted laterally. Identify in the skin fold between the gracilis and sartorius muscle where the pulsation of the superficial part of the medial saphenous artery disappears.

4. Make a longitudinal superficial skin incision cranial to this point, thereby avoiding inadvertently damaging the medial saphenous vessels.

5. Use a blunt-tip scissors to dissect the underlying subcutaneous tissue.

6. The fascia division of the sartorius and gracilis muscle is divided cranial to the penetration site of the medial saphenous vessels, first with a small blunt-tip surgical forceps and then digitally.

7. The two muscle groups are separated with a small self-retaining tissue retractor while taking care not to damage the femoral nerve and vessels.

8. The artery is isolated for a length of approximately 1 to $2 \mathrm{~cm}$ using blunt dissection.

9. Rotate the beveled venflon needle so the lumen is facing upwards. Bend the needle a little so it will follow the vessel curvature more easily. Remember to ensure the needle still can move back and forth with little effort.

10. The artery is punctured, the needle retracted and the Seldinger guide wire is inserted through the venflon tube.

11. Remove the venflon tube while gently applying pressure on the artery to ensure fixation of the guidewire.

12. The sheath with introducer is inserted over the Seldinger wire and advanced to the desired position, after which the introducer and Seldinger wire is removed.

13. The femoral vein is located just below and medial to the artery. After isolating the vein using blunt dissection, the vein is cannulated as previously described for the artery.

14. To verify that both the artery and vein are correctly catheterized, draw blood from both sheaths and compare the color of the samples. Whenever drawing a blood sample, remember to subsequently flush the catheters with heparinized saline in order to maintain catheter patency.

15. The catheters are secured and the skin is closed with a few sutures.

16. A vessel loop can be placed around the artery and vein in order to fixate the vessels prior to puncture. Despite its advantages, we generally avoid this additional step, because it requires excess dissection of the vessels and can increase the risk of damaging the lateral and deep branches.

\section{Transcardial perfusion}

1. The following equipment is needed for the procedure: perfusion system (Fig. 2), relevant perfusion fluids, scalpel, blunt-tip surgical scissors, bone shears, and two large self-holding forceps of which at least one has a curved tip. A self retaining sternal retractor is not absolutely necessary, but it eases the procedure considerably. 
2. Transcardial perfusion must be performed in a well ventilated room with a high power fume extraction system and facilities that allow collection of perfusate liquids and blood for subsequent safe disposal.

3. After intravenously injecting a lethal dose of pentobarbital, test the interdigital pain reflex before continuing.

4. The best access to the heart and aorta is provided via a median sternotomy. Make a deep longitudinal skin incision, extending from the manubrium to the xiphoid process of the sternum. Gain access to the thoracic cavity by making a small incision in the diaphragm just below the xiphoid process.

5. Insert the bone shears into the thoracic cavity and bisect the sternum. Care must be taken when performing this procedure, because the heart may be adherent at some place to the inside of the sternum. Therefore, make certain that the blade of the bone shears rests at all time inside the thoracic cavity against the inside of the sternum.

6. After the sternum has been divided, put in the self-retaining sternal retractor and open the thoracic cavity.

7. If still intact, make a small incision in the pericardium at the apex of the heart and then digitally open the pericardial sac.

8. Identify the left ventricle, the right auricle and the aorta together with the superior caval vein (Fig. 1C)

9. Make a small superficial cut near the apex of the heart and access the left ventricle by perforating the myocardium with a blunt-tipped instrument.

10. Insert the perfusion cannula inside the left ventricle and move the cannula tip cranially into the aorta.

11. When the cannula is felt between the fingers in the aorta, clamp the cannula into place with the self-holding curved forceps.

12. Make an incision in the right auricle and begin injecting the fixative through the perfusion cannula. The flow of perfusate in the aorta can be felt, and blood will pour out through the incised right auricle as the perfusate flow into the minipig vessels continues. Notice how systemic perfusion with paraformaldehyde induces hyperextension of the limbs and twitching of the superficial musculature due to aldehyde crosslinkage of nerves and muscles. When using a perfusion system powered by compressed air instead of gravity, make sure to remove the cannula, or clamp the clear silicone tube, before the canister is empty, in order to avoid air from entering the vascular system.
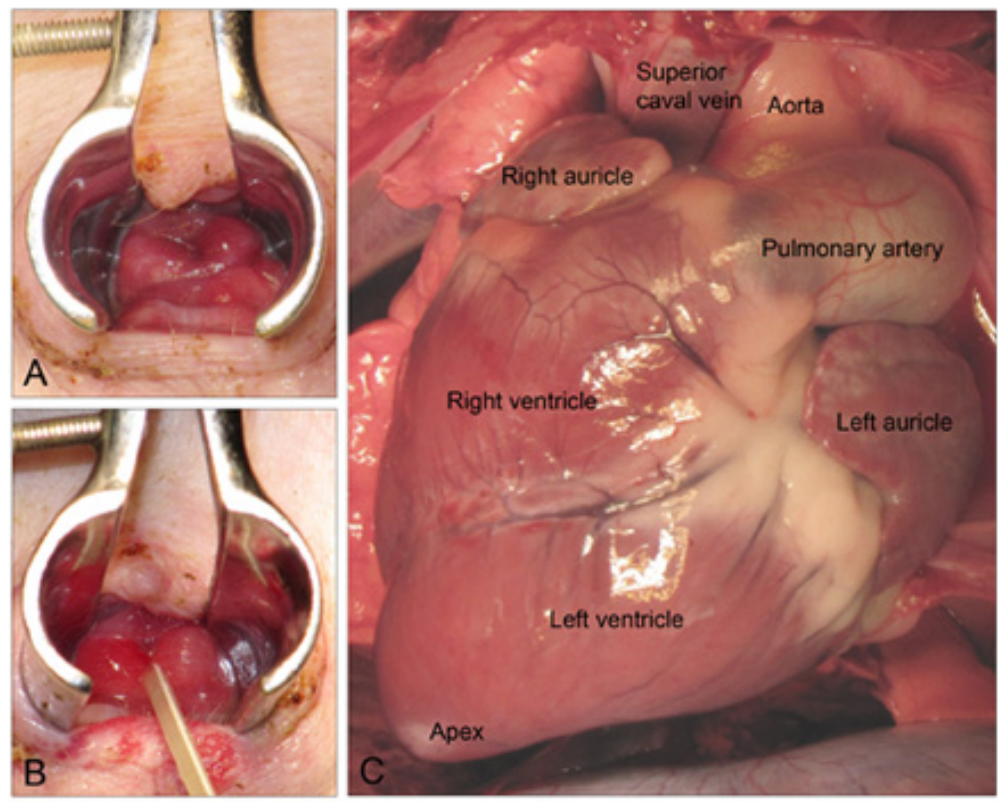

Figure 1. A: The female Göttingen minipig external urethral opening. B: The urethral opening after insertion of catheter. C: Ventral view of the minipig heart after median sternotomy. 

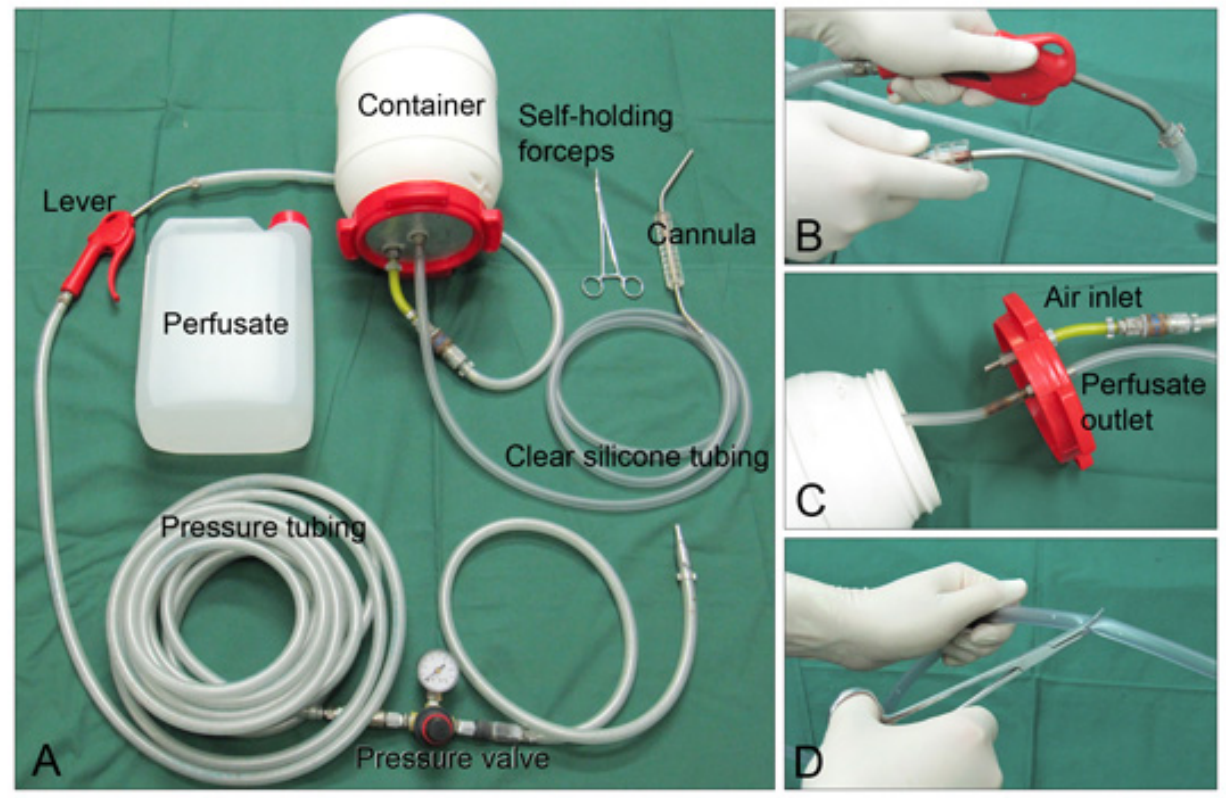

Figure 2. A: The perfusion system consists of inlet tubing with an adjustable pressure valve and a lever used to control inlet of compressed air into the container holding the perfusate. Clear silicone tubing connects the container to a blunt-tip cannula made from a $4 \mathrm{~mm}$ steel pipe. B and C: By compressing the inlet lever, the pressure rises in the container and the perfusate is propelled through the cannula. $\mathbf{D}$ : When the clear silicone tubing and cannula are filled with perfusate, the flow is stopped with a clamp and the system is ready for use.

\section{Discussion}

This presentation provides step-by-step visual instructions on how to perform four frequently used procedures in minipig research ${ }^{12,13}$. The techniques described may be used with little or no adaptation in landrace pigs and other large research animals such as dogs and sheep ${ }^{14}$. Aseptic technique must be employed to avoid infections, when performing the described procedures in survival studies.

Induction of anesthesia is important for animal welfare and scientific reasons, and should provide absence of corneal reflexes and good muscular relaxation, before endotracheal intubation is attempted. However, because the anaesthetics used for induction are not given in fixed doses, but administered until the desired effect is observed, it is important to closely monitor the animal due to the risk of apnoea, especially if using propofol $^{6,10}$. For tracheal intubation, the use of xylocaine spray or muscle relaxant can facilitate the introduction of the tube, but the use of neuromuscular blockers should only be done by personnel who are well-trained in such procedures so as to conform with ethical requirements. Care should be taken not to traumatize the laryngeal passage during intubation, in order to avoid tracheal swelling and edema. Airway edema can be treated with NSAID or similar anti-inflammatory drugs ${ }^{10}$. Pigs can be intubated from various positions, but having the animal on its back tends to be easier for those who regularly carry out human intubation, as it presents the airway in a similar manner ${ }^{6}$. The most common error during intubation is inadvertent placement of the tube in the esophagus, which is situated just below the laryngeal passage when the animal is placed on its back. Note, the tube should never be advanced without first clearly identifying the arytenoids cartilages and vocal cords.

Transurethral catheterization of the urinary bladder through the urethra is easily done in female pigs, while this procedure is extremely difficult, perhaps impossible, in males ${ }^{6}$. It should be noted that since the female urethral orifice is anterior to the vagina, the larger vaginal opening can be mistakenly catheterized. The use of sterile and disinfecting lubricant is advisable for survival studies. Furthermore, lubricant with local anesthetic effect may ease the catheterization procedure.

When performing femoral artery and vein catherization, the choice of vessel catheters and how they are secured differ between non-survival and survival studies, especially when catheters are implanted for an extended period of time ${ }^{6}$. For instance catheters for chronic implantation should be highly biocompatible, have retention beads or cuffs for fixation as well as not traumatize the vessel wall over time, thus inducing thrombosis or erosion ${ }^{6}$.

In the present video, we use a closed system powered by compressed air for transcardial perfusion. Another option is to use a system driven by gravity. The advantage of gravity systems in comparison to systems utilizing compressed air is that they are simple, efficient, and present no risk of pressurized air entering the vascular system. However, the hydrostatic pressure exerted by gravity systems changes continuously during perfusion, and this can affect the histological results ${ }^{15}$. Depending on which type of histopathological studies one wants to perform, it may be advantageous to perfuse the animal with heparinized saline first. This is particular importantance when performing immunofluorescent staining in order to avoid autofluorescence problems caused by erythrocytes. Furthermore, if the organ of interest is the brain, the descending aorta could be clamped to save fixative. However, in pigs, this step entails additional dissecting and will prolong the procedure. 


\section{Disclosures}

All procedures were approved by the Danish Experimental Animal Inspectorate, Danish Ministry of Justice and were conducted according to the

"Principles of laboratory animal care" (NIH publication No. 86-23, revised 1985).

\section{Acknowledgements}

The study was financially supported by the Lundbeck Foundation, The Karen Elise Jensen Foundation, The Health Research Fund of Central Denmark Region and The Institute of Clinical Medicine Aarhus University Hospital. We acknowledge with gratitude the skilful assistance of Donald F Smith, Anders Steen Soerensen, Henrik Soerensen, Asbjoern Ettrup-Christensen, and the staff at Paaskehoejgaard.

\section{References}

1. Lind, N.M., Moustgaard, A., Jelsing, J., Vajta, G., Cumming, P., Hansen, A.K. The use of pigs in neuroscience: modeling brain disorders. Neurosci Biobehav Rev 31 (5) : 728-751 (2007).

2. Ettrup, K.S., Sorensen, J.C., Bjarkam, C.R. The anatomy of the Gottingen minipig hypothalamus. J Chem Neuroanat 39 (3) : $151-165$ (2010)

3. Larsen, M.O., Rolin, B., Sturis, J., et al. Measurements of insulin responses as predictive markers of pancreatic beta-cell mass in normal and beta-cell-reduced lean and obese Gottingen minipigs in vivo. Am J Physiol Endocrinol Metab 290 (4) : E670-E677 (2006).

4. Forster, R., Ancian, P., Fredholm, M., Simianer, H., Whitelaw, B. The minipig as a platform for new technologies in toxicology. J Pharmacol Toxicol Methods (2010).

5. Popov, A.F., Dorge, H., Hinz, J. et al. Accelerated intimal hyperplasia in aortocoronary internal mammary vein grafts in minipigs. J Cardiothorac Surg $3: 20$ (2008).

6. Swindle, M.M. Swine in the Laboratory: Surgery, Anesthesia, Imaging, and Experimental Techniques. second ed. ed. CRC Press (2007).

7. Bjarkam, C.R., Cancian, G., Glud, A.N., Ettrup, K.S., Jorgensen, R.L., Sorensen, J.C. MRI-guided stereotaxic targeting in pigs based on a stereotaxic localizer box fitted with an isocentric frame and use of SurgiPlan computer-planning software. J Neurosci Methods 183 (2) : 119-126 (2009).

8. Val-Laillet, D., Blat, S., Louveau, I., Malbert, C.H. A computed tomography scan application to evaluate adiposity in a minipig model of human obesity. Br J Nutr 1-10 (2010).

9. Larsen, M., Bjarkam, C.R., Stoltenberg, M., Sorensen, J.C., Danscher, G. An autometallographic technique for myelin staining in formaldehyde-fixed tissue. Histol Histopathol 18 (4) : 1125-1130 (2003).

10. Alstrup, A.K.O. Anaesthesia and Analgesia in Ellegaard Göttingen minipigs. Jannerup.DK (2010).

11. Jensen, K.N., Deding, D., Sorensen, J.C., Bjarkam, C.R. Long-term implantation of deep brain stimulation electrodes in the pontine micturition centre of the Gottingen minipig. Acta Neurochir (Wien) 151 (7) : 785-794 (2009).

12. Cumming, P., Moller, M., Benda, K., et al. A PET study of effects of chronic 3,4-methylenedioxymethamphetamine (MDMA, "ecstasy") on serotonin markers in Gottingen minipig brain. Synapse 61 (7) : 478-487 (2007).

13. Olsen, A.K., Keiding, S., Munk, O.L. Effect of hypercapnia on cerebral blood flow and blood volume in pigs studied by positron emission tomography. Comp Med 56 (5) : 416-420 (2006).

14. Jonkers, B.W., Sterk, J.C., Wouterlood, F.G. Transcardial perfusion fixation of the CNS by means of a compressed-air-driven device. J Neurosci Methods 12 (2) : 141-149 (1984). 